\title{
Immunogenicity of 13-valent pneumococcal conjugate vaccine in immunocompetent older adults with stable underlying medical conditions
}

Beate Schmoele-Thoma 1,*, Lisa A. Jackson², Richard N. Greenberg ${ }^{3}$, Robert Frenck ${ }^{4}$, Alejandra Gurtman ${ }^{5}$, Vani Sundaraiyer ${ }^{6}$, William C. Gruber ${ }^{5}$, Daniel A. Scott ${ }^{5}$ and Raul E. Isturiz ${ }^{7}$

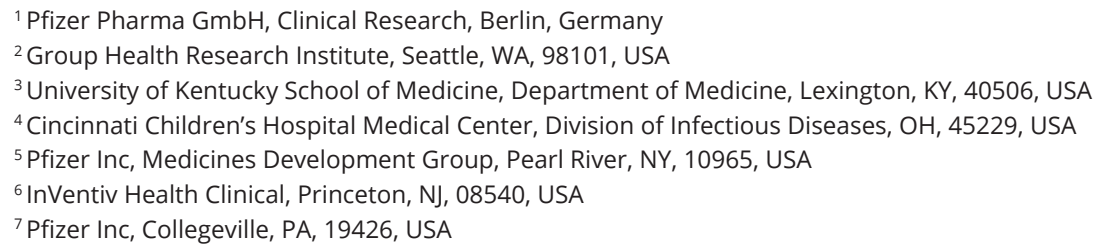

\begin{abstract}
The 13-valent pneumococcal conjugate vaccine (PCV13) elicits robust functional antibody responses in immunocompetent adults aged $\geq 50$ years, including healthy adults and those considered at risk (ie, stable, chronic cardiovascular disease, pulmonary disease, or diabetes mellitus). This analysis is consistent with data suggesting immunocompetent at-risk populations aged $\geq 50$ years produce functional antipneumococcal antibody responses to PCV13 comparable to those not at risk. An OPA titer that correlates with protection has not been defined for adults; however, PCV13 elicits generally higher responses than the 23-valent pneumococcal polysaccharide vaccine (PPSV23) one month after vaccination. PCV13 vaccination of immunocompetent at-risk populations likely results in benefits analogous to those without additional risk.
\end{abstract}

Keywords: 13-valent pneumococcal conjugate vaccine; at-risk adults; pneumococcal disease; diabetes mellitus; cardiovascular disease; pulmonary disease

\section{Introduction}

Streptococcus pneumoniae is a major cause of morbidity and mortality worldwide [1]. Adults $\geq 50$ years of age are at increased risk for developing invasive pneumococcal disease (IPD) and community-acquired pneumonia (CAP) $[2,3]$. In addition, underlying chronic medical conditions such as cardiovascular disease, pulmonary disease, and diabetes mellitus, all of which have been shown to increase the risk for pneumococcal pneumonia, are more prevalent in individuals $>50$ years of age [4]. An analysis of data from the Active Bacterial Core surveillance system in the United States reported that among cases of IPD in subjects 50 to 64 and $\geq 65$ years of age, there was a disproportionately high frequency of cardiac disease $(12 \%$ and $37 \%$, respectively), chronic lung disease (21\% and $31 \%)$, and diabetes (22\% and 25\%) [5]. Thus, effective strategies to prevent pneumococcal disease in these at-risk individuals are of great importance.

Two previous studies assessed safety and immunogenicity of the 13-valent pneumococcal conjugate vaccine (PCV13) in healthy, pneumococcal vaccine-naive adults 50 to 64 years of age and a third study assessed safety and immunogenicity of PCV13 in adults $\geq 70$ years of age who were previously vaccinated with 1 dose of 23-valent pneumococcal polysaccharide vaccine (PPSV23). All 3 studies allowed enrollment of subjects with stable chronic underlying diseases such as chronic cardiovascular disease, pulmonary disease, or diabetes mellitus. These studies showed that PCV13 elicits robust functional antibody responses in a population that included these atrisk subjects [6-8]. We now report a post hoc analysis of the 3 previously published studies comparing functional antibody responses to PCV13 in at-risk adults $\geq 50$ years of

*Corresponding author: Beate Schmoele-Thoma, MD, Senior Director Clinical Research, Pfizer Pharma GmbH, Linkstraße 10, Berlin, Germany. Tel.: +49 817799 7937; Fax: +49 8177 997938; Email: beate.schmoelethoma@pfizer.com

Received 27 March 2015 Revised 1 June 2015 Accepted 10 June 2015 Published 18 June 2015

Citation: Schmoele-Thoma B, Jackson LA, Greenberg RN, Frenck R, Gurtman A, Sundaraiyer V, Gruber WC, Scott DA, Isturiz RE. Immunogenicity of 13valent pneumococcal conjugate vaccine in immunocompetent older adults with stable underlying medical conditions. J Vaccines Immun. 2015; 3(2):712. DOI:10.14312/2053-1273.2015-2

Copyright: (c) 2015 Schmoele-Thoma B, et al. Published by NobleResearch Publishers. This is an open-access article distributed under the terms of the Creative Commons Attribution License, which permits unrestricted use, distribution and reproduction in any medium, provided the original author and source are credited. 
age with responses in those considered not at increased risk. In addition, inclusion of PCV13 and PPSV23 study groups in 2 studies permits a similar post hoc comparison of functional antibody responses to vaccination in pneumococcal vaccine-naive at-risk subjects 60 to 64 years of age.

\section{Materials and methods}

\section{Study design}

At-risk subjects were defined as those diagnosed with stable chronic cardiovascular, pulmonary, liver, or renal disease, or diabetes mellitus (type 1 or 2). Stable disease, which was part of inclusion/exclusion criteria for each study, was defined as not requiring significant change in therapy or hospitalization for worsening disease within 12 weeks before vaccination. The most common diagnoses for cardiovascular disease were coronary artery disease, angina pectoris, and congestive cardiac failure; for pulmonary disease, the most common diagnoses were asthma, chronic obstructive pulmonary disease, and chronic bronchitis.

Functional antibody responses in participants of the 3 previous randomized trials who were vaccinated with PCV13 or PPSV23 have been reported [6-8]. Study 1 enrolled 470 PPSV23-naive adults aged 60 to 64 years (median age, $61 \mathrm{y}), 125$ of whom were considered to be at risk [6]. Study 2 enrolled 799 PPSV23-naive adults aged 50 to 64 years (cohort 1, 60 to 64 years [median age, $62 \mathrm{y}$ ] and cohort 2, 50 to 59 years [median age, $54 \mathrm{y}$ ]), 148 of whom were considered to be at risk [7]. Study 3 enrolled 431 adults aged $\geq 70$ years (median age, $76 \mathrm{y}$ ) who were vaccinated with PPSV23 $\geq 5$ years before study enrollment, 153 of whom were considered to be at risk [8]. Blood samples in the 3 studies were drawn for immunogenicity analyses before and approximately 1 month after vaccination [6-8].

\section{Vaccines}

PCV13 (Prevnar 13/Prevenar $13^{\circledR}$, Pfizer Inc) contains polysaccharides of pneumococcal serotypes 1, 3, 4, 5, 6A, 6B, 7F, 9V, 14, 18C, 19F, 19A, and 23F individually conjugated to a nontoxic form of diphtheria toxin crossreactive material 197. Each $0.5-\mathrm{mL}$ dose contains $2.2 \mu \mathrm{g}$ of each serotype, except type 6B, which is included at $4.4 \mu \mathrm{g}$. PPSV23 (Pneumovax 23 ${ }^{\circledR}$, Merck \& Company, Inc) consists of a capsular polysaccharide from 12 of the serotypes included in PCV13 (all except 6A), as well as 11 additional serotypes $(2,8,9 \mathrm{~N}, 10 \mathrm{~A}, 11 \mathrm{~A}, 12 \mathrm{~F}, 15 \mathrm{~B}, 17 \mathrm{~F}, 20,22 \mathrm{~F}$, and $33 F)$. The vaccine is formulated to contain $25 \mu \mathrm{g}$ of each of the 23 polysaccharide serotypes per $0.5-\mathrm{mL}$ dose.

\section{Immunogenicity analysis}

Opsonophagocytic assay (OPA) geometric mean titers (GMTs) approximately 1 month after PCV13 administration in at-risk subjects $\geq 50$ years of age with cardiovascular disease, pulmonary disease, or diabetes mellitus were descriptively compared with those in subjects without these underlying medical conditions. In addition, in studies 1 and 2, OPA GMTs after PCV13 or PPSV23 vaccination were compared in at-risk subjects 60 to 64 years of age with cardiovascular disease, pulmonary disease, or diabetes mellitus previously naive to pneumococcal vaccination
$[6,7]$. To increase sample sizes of the at-risk population in these 2 studies, OPA data from study participants were pooled; study 3 was not included in this pooled analysis because adults enrolled into this study differed in age and immunization status [8].

\section{Statistical analysis}

OPA GMTs elicited by PCV13 and PPSV23 were calculated for each serotype; comparisons were made between at-risk subjects and subjects not considered at risk. Ratio of GMTs for PCV13 to PPSV23, which included all subjects with a determinate OPA titer to the given serotype, were calculated by back-transforming the mean difference between vaccine groups on the logarithmic scale; confidence intervals (Cls) for the ratio are back-transformations of a $\mathrm{Cl}$ based on the Student $t$ distribution for the mean difference of the logarithms of the measures (PCV13 - PPSV23). Geometric mean fold rise (GMFR) was calculated using OPA GMT data for all subjects with available data from both the pre- and postvaccination blood draws; Cls are back-transformations of a $\mathrm{Cl}$ based on the Student $t$ distribution for the mean fold rise. A statistically significantly higher GMT was inferred when the lower limit of the 2-sided $95 \% \mathrm{Cl}$ for the GMFR was $>1$. Similar criteria were used for GMT ratios.

\section{Results}

\section{Baseline characteristics}

Mean age across the 3 studies ranged from 54 to 77 years. The majority of subjects in all studies were white and nonHispanic. The predominant at-risk condition was diabetes in study 1 , pulmonary disease in study 2 , and cardiovascular disease in study 3 . Subjects in the clinical studies were not stratified for risk conditions at enrollment; therefore, number of subjects in the at-risk groups varied, and in some cases, was very low. The percentage of subjects without underlying medical conditions ranged from 65\% to $83 \%$ across the 3 studies (Supplementary Table). Mean time since prior PPSV23 vaccination in study 3 was 8.4 years (range, 5.0-17.1).

\section{Immunogenicity}

Across all 3 studies, OPA GMTs to PCV13 serotypes 1 month after vaccination were comparable in at-risk subjects with stable chronic medical conditions and those without those conditions (Supplementary Figure), despite slight variations between studies, at-risk condition, and serotypes. In a pooled analysis of data from studies 1 and 2 evaluating subjects 60 to 64 years of age previously naive to pneumococcal vaccination who were diagnosed with cardiovascular disease, pulmonary disease, or diabetes mellitus, OPA GMTs increased from before to after PCV13 vaccination for the 12 serotypes common to both vaccines and serotype 6A (Table 1). GMFRs from prevaccination to postvaccination were generally higher among PCV13 than PPSV23 recipients for all serotypes and risk conditions. PCV13 elicited numerically higher OPA GMTs compared with PPSV23 for the majority of serotypes in all at-risk groups. Statistically significantly higher OPA GMTs were observed for 4, 9 and 7 of the 12 common serotypes in subjects with cardiovascular disease, pulmonary disease, or diabetes mellitus, respectively, and in all at-risk groups for serotype 6A, a serotype unique to PCV13 (Table 2). 
Table 1 OPA GMTs and GMFRs in PPSV23-naive subjects 60-64 years of age with at-risk conditions in studies 1 and 2 (Cohort 1 ).

\begin{tabular}{|c|c|c|c|c|c|c|c|}
\hline & \multirow{3}{*}{ Serotype } & \multicolumn{2}{|c|}{ Cardiovascular disease } & \multicolumn{2}{|c|}{ Pulmonary disease } & \multicolumn{2}{|c|}{ Diabetes mellitus } \\
\hline & & PCV13 & PPSV23 & PCV13 & PPSV23 & PCV13 & PPSV23 \\
\hline & & $n^{a}=43-52$ & $n^{a}=32-43$ & $n^{a}=63-78$ & $n^{a}=36-47$ & $n^{a}=84-105$ & $n^{a}=60-82$ \\
\hline \multirow{2}{*}{1} & Postvaccination $\mathrm{GMT}^{\mathrm{b}}$ & 139 & 102 & 140 & 61 & 189 & 116 \\
\hline & GMFR $(95 \% \mathrm{Cl})^{\mathrm{c}}$ & $\begin{array}{c}20.1 \\
(12.20-33.05)\end{array}$ & $\begin{array}{c}11.8 \\
(6.93-19.98)\end{array}$ & $\begin{array}{c}22.7 \\
(14.72-34.91)\end{array}$ & $\begin{array}{c}10.0 \\
(5.49-18.28)\end{array}$ & $\begin{array}{c}30.1 \\
(21.58-42.01)\end{array}$ & $\begin{array}{c}19.9 \\
(13.86-28.42)\end{array}$ \\
\hline \multirow[t]{3}{*}{3} & Prevaccination GMT & 5 & 7 & 6 & 6 & 6 & 7 \\
\hline & Postvaccination $\mathrm{GMT}^{\mathrm{b}}$ & 74 & 69 & 67 & 50 & 80 & 84 \\
\hline & GMFR $(95 \% \mathrm{CI})^{c}$ & $\begin{array}{c}14.3 \\
(9.44-21.67)\end{array}$ & $\begin{array}{c}10.3 \\
(6.83-15.65)\end{array}$ & $\begin{array}{c}10.4 \\
(7.24-14.85)\end{array}$ & $\begin{array}{c}8.3 \\
(5.43-12.82)\end{array}$ & $\begin{array}{c}14.2 \\
(10.28-19.67)\end{array}$ & $\begin{array}{c}11.2 \\
(8.20-15.32)\end{array}$ \\
\hline \multirow[t]{3}{*}{4} & Prevaccination GMT & 15 & 14 & 16 & 11 & 31 & 12 \\
\hline & Postvaccination GMT ${ }^{\mathrm{b}}$ & 1502 & 1145 & 1746 & 341 & 2597 & 639 \\
\hline & $\operatorname{GMFR}(95 \% \mathrm{CI})^{\mathrm{c}}$ & $\begin{array}{c}98.7 \\
(42.34-230.27)\end{array}$ & $\begin{array}{c}83.8 \\
(30.70-228.68)\end{array}$ & $\begin{array}{c}109.1 \\
(54.85-217.16)\end{array}$ & $\begin{array}{c}30.2 \\
(11.23-81.43)\end{array}$ & $\begin{array}{c}84.0 \\
(44.89-157.13)\end{array}$ & $\begin{array}{c}52.2 \\
(25.77-105.90)\end{array}$ \\
\hline \multirow[t]{3}{*}{5} & Prevaccination GMT & 6 & 5 & 6 & 6 & 6 & 7 \\
\hline & Postvaccination $\mathrm{GMT}^{\mathrm{b}}$ & 344 & 152 & 213 & 91 & 251 & 153 \\
\hline & GMFR $(95 \% \mathrm{CI})^{\mathrm{c}}$ & $\begin{array}{c}54.2 \\
(30.51-96.29)\end{array}$ & $\begin{array}{c}31.1 \\
(16.28-59.29)\end{array}$ & $\begin{array}{c}35.2 \\
(21.91-56.51)\end{array}$ & $\begin{array}{c}16.4 \\
(8.34-32.35)\end{array}$ & $\begin{array}{c}44.8 \\
(29.29-68.57)\end{array}$ & $\begin{array}{c}22.7 \\
(14.43-35.72)\end{array}$ \\
\hline \multirow[t]{3}{*}{$6 A^{d}$} & Prevaccination GMT & 13 & 17 & 25 & 13 & 21 & 14 \\
\hline & Postvaccination $\mathrm{GMT}^{\mathrm{b}}$ & 2110 & 337 & 1984 & 98 & 2104 & 222 \\
\hline & GMFR $(95 \% \mathrm{CI})^{c}$ & $\begin{array}{c}166.8 \\
(80.98-343.75)\end{array}$ & $\begin{array}{c}20.4 \\
(8.83-47.10)\end{array}$ & $\begin{array}{c}78.5 \\
(43.37-142.23)\end{array}$ & $\begin{array}{c}7.6 \\
(3.46-16.62)\end{array}$ & $\begin{array}{c}98.8 \\
(57.59-169.35)\end{array}$ & $\begin{array}{c}15.5 \\
(8.73-27.53)\end{array}$ \\
\hline \multirow[t]{3}{*}{$6 \mathrm{~B}$} & Prevaccination GMT & 37 & 36 & 40 & 19 & 39 & 32 \\
\hline & Postvaccination $\mathrm{GMT}^{\mathrm{b}}$ & 1666 & 1065 & 1196 & 425 & 1250 & 711 \\
\hline & GMFR $(95 \% \mathrm{CI})^{c}$ & $\begin{array}{c}44.5 \\
(19.03-104.20)\end{array}$ & $\begin{array}{c}29.7 \\
(12.76-69.35)\end{array}$ & $\begin{array}{c}30.1 \\
(15.24-59.29)\end{array}$ & $\begin{array}{c}21.9 \\
(8.93-53.78)\end{array}$ & $\begin{array}{c}32.1 \\
(17.52-58.94)\end{array}$ & $\begin{array}{c}22.2 \\
(11.83-41.82)\end{array}$ \\
\hline $7 F$ & Prevaccination GMT & 8 & 5 & 6 & 7 & 7 & 7 \\
\hline \multirow[t]{3}{*}{$9 \mathrm{~V}$} & Prevaccination GMT & 10 & 12 & 17 & 10 & 12 & 12 \\
\hline & Postvaccination $\mathrm{GMT}^{\mathrm{b}}$ & 740 & 190 & 952 & 84 & 768 & 163 \\
\hline & GMFR $(95 \% \mathrm{CI})^{\mathrm{c}}$ & $\begin{array}{c}72.8 \\
(32.82-161.65)\end{array}$ & $\begin{array}{c}15.4 \\
(6.00-39.60)\end{array}$ & $\begin{array}{c}55.9 \\
(29.29-106.66)\end{array}$ & $\begin{array}{c}8.3 \\
(3.39-20.52)\end{array}$ & $\begin{array}{c}62.9 \\
(35.16-112.60)\end{array}$ & $\begin{array}{c}13.8 \\
(6.45-29.60)\end{array}$ \\
\hline \multirow[t]{3}{*}{14} & Prevaccination GMT & 38 & 39 & 25 & 64 & 27 & 32 \\
\hline & Postvaccination $\mathrm{GMT}^{\mathrm{b}}$ & 853 & 991 & 600 & 442 & 538 & 606 \\
\hline & GMFR $(95 \% \mathrm{CI})^{\mathrm{c}}$ & $\begin{array}{c}22.3 \\
(9.95-49.99)\end{array}$ & $\begin{array}{c}25.6 \\
(10.50-62.62)\end{array}$ & $\begin{array}{c}24.1 \\
(12.39-46.79)\end{array}$ & $\begin{array}{c}6.9 \\
(3.42-14.00)\end{array}$ & $\begin{array}{c}20.1 \\
(11.22-36.02)\end{array}$ & $\begin{array}{c}19.1 \\
(10.56-34.59)\end{array}$ \\
\hline \multirow[t]{3}{*}{$18 \mathrm{C}$} & Prevaccination GMT & 19 & 28 & 27 & 29 & 20 & 37 \\
\hline & Postvaccination $\mathrm{GMT}^{\mathrm{b}}$ & 2186 & 1245 & 1610 & 616 & 1716 & 1001 \\
\hline & GMFR $(95 \% \mathrm{CI})^{c}$ & $\begin{array}{c}117.2 \\
(59.00-232.80)\end{array}$ & $\begin{array}{c}44.3 \\
(21.86-89.58)\end{array}$ & $\begin{array}{c}60.4 \\
(31.02-117.70)\end{array}$ & $\begin{array}{c}21.5 \\
(9.13-50.53)\end{array}$ & $\begin{array}{c}86.7 \\
(51.17-146.86)\end{array}$ & $\begin{array}{c}27.0 \\
(15.59-46.68)\end{array}$ \\
\hline \multirow[t]{3}{*}{$19 \mathrm{~A}$} & Prevaccination GMT & 21 & 18 & 18 & 20 & 22 & 29 \\
\hline & Postvaccination $\mathrm{GMT}^{\mathrm{b}}$ & 662 & 368 & 558 & 261 & 669 & 364 \\
\hline & GMFR $(95 \% \mathrm{Cl})^{c}$ & $\begin{array}{c}31.5 \\
(17.82-55.53)\end{array}$ & $\begin{array}{c}20.4 \\
(11.76-35.25)\end{array}$ & $\begin{array}{c}30.5 \\
(18.90-49.08)\end{array}$ & $\begin{array}{c}12.9 \\
(7.19-23.17)\end{array}$ & $\begin{array}{c}30.4 \\
(20.59-44.80)\end{array}$ & $\begin{array}{c}12.5 \\
(8.27-18.77)\end{array}$ \\
\hline \multirow[t]{3}{*}{$19 \mathrm{~F}$} & Prevaccination GMT & 22 & 14 & 12 & 11 & 16 & 27 \\
\hline & Postvaccination $\mathrm{GMT}^{\mathrm{b}}$ & 854 & 476 & 407 & 447 & 818 & 647 \\
\hline & GMFR $(95 \% \mathrm{CI})^{c}$ & $\begin{array}{c}39.2 \\
(19.65-78.10)\end{array}$ & $\begin{array}{c}32.9 \\
(14.98-72.15)\end{array}$ & $\begin{array}{c}32.7 \\
(19.14-55.92)\end{array}$ & $\begin{array}{c}40.6 \\
(19.06-86.56)\end{array}$ & $\begin{array}{c}52.2 \\
(32.28-84.46)\end{array}$ & $\begin{array}{c}24.2 \\
(14.24-41.01)\end{array}$ \\
\hline \multirow[t]{3}{*}{$23 \mathrm{~F}$} & Prevaccination GMT & 6 & 9 & 6 & 5 & 8 & 8 \\
\hline & Postvaccination $\mathrm{GMT}^{\mathrm{b}}$ & 196 & 49 & 264 & 28 & 309 & 40 \\
\hline & GMFR $(95 \% \mathrm{CI})^{\mathrm{c}}$ & $\begin{array}{c}35.7 \\
(17.29-73.60) \\
\end{array}$ & $\begin{array}{c}5.8 \\
(3.01-11.00) \\
\end{array}$ & $\begin{array}{c}47.5 \\
(28.25-79.80) \\
\end{array}$ & $\begin{array}{c}6.1 \\
(2.86-12.88) \\
\end{array}$ & $\begin{array}{c}37.9 \\
(22.33-64.33) \\
\end{array}$ & $\begin{array}{c}5.0 \\
(2.94-8.39) \\
\end{array}$ \\
\hline
\end{tabular}

Abbreviations: GMT=geometric mean titer; GMFR=geometric mean fold rise; OPA=opsonophagocytic assay; PCV13=13-valent pneumococcal conjugate vaccine; PPSV23=23-valent pneumococcal polysaccharide vaccine; ${ }^{a}$ Number of subjects with valid and determinate OPA results for the specified serotype at both the prevaccination and the postvaccination blood draws; ${ }^{b}$ One month postvaccination; ' $\mathrm{GMFRs}$ were calculated using all subjects with available data from both the prevaccination and the postvaccination blood draws. Cls are back-transformations of a $\mathrm{Cl}$ based on the Student $t$ distribution for the mean fold rise; 'Serotype 6A is uniquely present in PCV13.

Note: GMTs were calculated using all subjects with available data for both the specified blood draws. 
Table 2 Comparison of pneumococcal OPA GMTs for PCV13 relative to PPSV23 in PPSV23-naive subjects 60-64 years of age with at-risk conditions in studies 1 and 2 (Cohort 1).

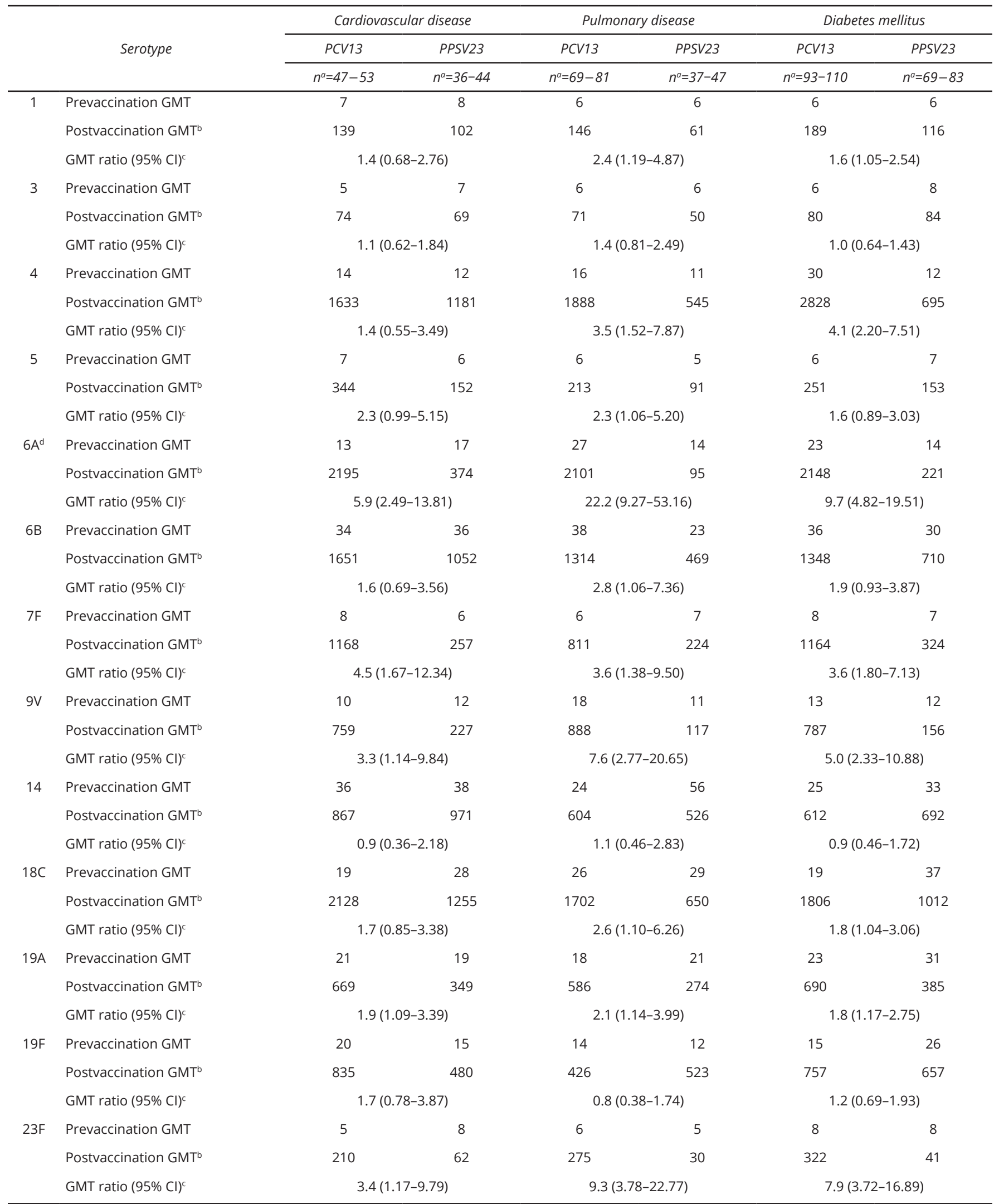

Abbreviations: GMT=geometric mean titer; OPA=opsonophagocytic assay; PCV13=13-valent pneumococcal conjugate vaccine; PPSV23=23-valent pneumococcal polysaccharide vaccine; a Number of subjects with a valid and determinate OPA result for a specific serotype; ${ }^{b}$ One month postvaccination; 'Ratio of postvaccination GMTs PCV13 to PPSV23 is calculated by back-transforming the mean difference between vaccine groups on the logarithmic scale. Cls for the ratio are back-transformations of a $\mathrm{Cl}$ based on the Student $t$ distribution for the mean difference of the logarithms of the measures (postvaccination PCV13 - postvaccination PPSV23); dSerotype 6A is uniquely present in PCV13.

Note: GMTs were calculated using all subjects with available data for the specified blood draw. 


\section{Discussion}

Previous studies have established an increased risk for pneumococcal disease in immunocompetent subjects with underlying chronic diseases [4, 5, 9-12], emphasizing the need for effective preventive strategies for these atrisk populations. Several studies have examined the responses to PCV or PPSV vaccination in at-risk adults [1319]; to date, no studies specifically examined responses after PCV13 vaccination in at-risk adults. Therefore, in this analysis, we evaluated data from 3 previous studies in immunocompetent PPSV23-naive and PPSV23preimmunized subjects $\geq 50$ years of age that included a considerable percentage of subjects with stable chronic underlying conditions known to put these subjects at increased risk for pneumococcal disease. An OPA titer that correlates with protection has not been defined for adults.

As shown in this study, PCV13 administered to adults with cardiovascular disease, pulmonary disease, or diabetes mellitus, elicited functional pneumococcal antibody responses that were generally comparable to those of individuals considered not to be at risk, regardless of previous PPSV23 administration. In PPSV23-naive adults 60 to 64 years of age with an at-risk condition, OPA GMTs increased from prevaccination to postvaccination with PCV13 or PPSV23 for the 12 serotypes common to both vaccines and 6A. GMFRs from prevaccination to postvaccination were higher for PCV13 than for PPSV23 with few exceptions. In general, PCV13 elicited higher responses than PPSV23 in these at-risk subjects, a number of which were statistically significant. This is consistent with the higher PCV13 responses observed in the primary studies comprising subjects with and without risk conditions $[6,7]$.

The limitations of this analysis lie in its descriptive nature and relatively low numbers of some at-risk groups, as subjects in the clinical studies were not stratified for risk conditions at enrollment; this resulted in variable numbers of subjects in each at-risk group, with low representation of some at-risk conditions. Although the original studies assessed antibody responses one year after vaccination, one of the studies evaluated responses in a subset of less than 100 subjects, which limits drawing meaningful conclusions; therefore, one year data were not included in this analysis. However, recent data from the Community Acquired Pneumonia Immunization Trial in Adults (CAPiTA) 65 years of age and older in the Netherlands, that included approximately 85,000 subjects, demonstrated that PCV13 compared to placebo is efficacious against vaccine-type CAP, including nonbacteremic CAP, as well as vaccine-type IPD; importantly, this study included immunocompetent subjects with chronic conditions such as cardiovascular disease, pulmonary disease, and diabetes mellitus [20, 21]. Antibody responses were assessed in a subset of subjects at 1, 12 and 24 months after PCV13 vaccination. Consistent with the observations in the present studies, functional antibody responses were similar across all evaluated underlying medical conditions and all serotypes. Furthermore, antibody levels remained above baseline across all evaluated time points [22].

\section{Conclusion}

The results presented herein suggest that vaccination of immunocompetent at-risk populations with PCV13 likely results in immunologic benefits analogous to those experienced by populations not at additional risk.

\section{Acknowledgments}

The authors would like to thank the investigators of the 3 studies (6115A1-004, -3010, and -3005) and Pinaki Biswas of Pfizer Inc and James Trammel and colleagues at inVentiv Health Clinical, LLC, for support with the data analyses. The assistance of the following research team members at the University of Kentucky who participated in essential study activities is appreciated: Beth Plummer, Malissia Van Hook, Debbie Plummer, Heather Flynn, Connie Gerardot, Dana Hargis, and Steve Sitzlar.

\section{Funding}

This study was supported by Pfizer Inc. Editorial/medical writing support was provided by Nicole Gudleski O'Regan, PhD, of Complete Healthcare Communications, Inc. and was funded by Pfizer Inc.

\section{Conflicts of Interest}

RNG, RF, and LAJ received funding from Pfizer to conduct this study and support from Pfizer for attendance at a scientific meeting. The University of Kentucky was supported in part by a National Institutes of Health research grant to their Aging Center (P30AG028383) and has received research grants from Viropharma, T2, PaxVax, and Bavarian-Nordic. BS-T, AG, WCG, DAS, and REI are employees of Pfizer Inc. VS is an employee of a Pfizer-contracted company.

\section{Supplementary data}

Supplementary data associated with this article can be found, at http://nobleresearch.org/Doi/10.14312/20531273.2015-2. These data include supplementary Table and Figure.

\section{References}

[1] World Health Organization. Pneumococcal disease. Accessed July 14 2015 at: http://www.who.int/ith/diseases/pneumococcal/en/.

[2] File TM Jr, Marrie TJ. Burden of community-acquired pneumonia in North American adults. Postgrad Med. 2010; 122(2):130-141.

[3] Centers for Disease Control and Prevention. ABCs Report: Streptococcus pneumoniae, 2010. Active Bacterial Core surveillance (ABCs): Emerging Infections Program Network. Accessed July 14, 2015 at: http://www. cdc.gov/abcs/reports-findings/survreports/spneu10.html.

[4] van Hoek AJ, Andrews N, Waight PA, Stowe J, Gates P, et al. The effect of underlying clinical conditions on the risk of developing invasive pneumococcal disease in England. J Infect. 2012; 65(1):17-24.

[5] Muhammad RD, Oza-Frank R, Zell E, Link-Gelles R, Narayan KM, et al. Epidemiology of invasive pneumococcal disease among high-risk adults since the introduction of pneumococcal conjugate vaccine for children. Clin Infect Dis. (2013); 56(5):e59-67.

[6] Greenberg RN, Gurtman A, Frenck RW, Strout C, Jansen KU, et al. Sequential administration of 13-valent pneumococcal conjugate vaccine and 23-valent pneumococcal polysaccharide vaccine in pneumococcal vaccine-naive adults 60-64 years of age. Vaccine. 2014; 32(20):2364-2374.

[7] Jackson LA, Gurtman A, van Cleeff M, Jansen KU, Jayawardene D, et al. Immunogenicity and safety of a 13-valent pneumococcal conjugate vaccine compared to a 23-valent pneumococcal polysaccharide vaccine in pneumococcal vaccine-naive adults. Vaccine. 2013; 31(35):35773584. 
[8] Jackson LA, Gurtman A, Rice K, Pauksens K, Greenberg RN, et al. Immunogenicity and safety of a 13-valent pneumococcal conjugate vaccine in adults 70 years of age and older previously vaccinated with 23-valent pneumococcal polysaccharide vaccine. Vaccine. 2013; 31(35):3585-3593.

[9] Ma HM, Tang WH, Woo J. Predictors of in-hospital mortality of older patients admitted for community-acquired pneumonia. Age Ageing. 2011; 40(6):736-741.

[10] Ricketson LJ, Nettel-Aguirre A, Vanderkooi OG, Laupland KB, Kellner JD. Factors influencing early and late mortality in adults with invasive pneumococcal disease in Calgary, Canada: a prospective surveillance study. PLoS ONE. 2013; 8(10):e71924.

[11] Ishiguro T, Takayanagi N, Yamaguchi S, Yamakawa H, Nakamoto K, et al. Etiology and factors contributing to the severity and mortality of community-acquired pneumonia. Intern Med. 2013; 52(3):317-324.

[12] Shea KM, Edelsberg J, Weycker D, Farkouh RA, Strutton DR, et al. Rates of pneumococcal disease in adults with chronic medical conditions. Open Forum Infect Dis. 2014; 1(1):ofu024.

[13] Simberkoff MS, Cross AP, Al-lbrahim M, Baltch AL, Geiseler PJ, et al. Efficacy of pneumococcal vaccine in high-risk patients. Results of a Veterans Administration Cooperative Study. N Engl J Med. 1986; 315(21):1318-1327.

[14] Butler JC, Breiman RF, Campbell JF, Lipman HB, Broome CV, et al. Pneumococcal polysaccharide vaccine efficacy. An evaluation of current recommendations. JAMA. 1993; 270(15):1826-1831.

[15] Dransfield MT, Nahm MH, Han MK, Harnden S, Criner GJ, et al. Superior immune response to protein-conjugate versus free pneumococcal polysaccharide vaccine in chronic obstructive pulmonary disease. Am J Respir Crit Care Med. 2009; 180(6):499-505.

[16] Musher DM, Rueda AM, Nahm MH, Graviss EA, Rodriguez-Barradas MC. Initial and subsequent response to pneumococcal polysaccharide and protein-conjugate vaccines administered sequentially to adults who have recovered from pneumococcal pneumonia. J Infect Dis. 2008; 198(7):1019-1027.

[17] Ridda I, Macintyre CR, Lindley R, Gao Z, Sullivan JS, et al. Immunological responses to pneumococcal vaccine in frail older people. Vaccine. 2009; 27(10):1628-1636.

[18] Vila-Corcoles A, Ochoa-Gondar O, Guzman JA, Rodriguez-Blanco $T$, Salsench $E$, et al. Effectiveness of the 23-valent polysaccharide pneumococcal vaccine against invasive pneumococcal disease in people 60 years or older. BMC Infect Dis. 2010; 10:73.

[19] Musher DM, Manoff SB, McFetridge RD, Liss CL, Marchese RD, et al. Antibody persistence ten years after first and second doses of 23valent pneumococcal polysaccharide vaccine, and immunogenicity and safety of second and third doses in older adults. Hum Vaccin. 2011; 7(9):919-928.

[20] Tomczyk S, Bennett NM, Stoecker C, Gierke R, Moore MR, et al. Use of 13-valent pneumococcal conjugate vaccine and 23-valent pneumococcal polysaccharide vaccine among adults aged $\geq 65$ years: recommendations of the Advisory Committee on Immunization Practices (ACIP). MMWR Morb Mortal Wkly Rep. 2014; 63(37):822-825.

[21] Bonten MJ, Huijts SM, Bolkenbaas M, Webber C, Patterson S, et al. Polysaccharide conjugate vaccine against pneumococcal pneumonia in adults. N Engl J Med. 2015; 372(12):1114-1125.

[22] Webber C, Patton M, Patterson S, Schmoele-Thoma B, Huijts S, et al. Exploratory efficacy endpoints in the community-acquired pneumonia immunization trial in adults (CAPiTA). Presented at: European Society of Clinical Microbiology and Infectious Diseases, April 25-28, 2015, Copenhagen, Denmark. 